\title{
Red blood cell deformability is diminished in patients with Chronic Fatigue Syndrome
}

\author{
Amit K. Saha ${ }^{\mathrm{a}, \mathrm{b}}$, Brendan R. Schmidt ${ }^{\mathrm{a}}$, Julie Wilhelmy ${ }^{\mathrm{b}}$, Vy Nguyen ${ }^{\mathrm{a}}$, Abed Abugherir ${ }^{\mathrm{a}}$, \\ Justin K. Do ${ }^{\mathrm{a}}$, Mohsen Nemat-Gorgani ${ }^{\mathrm{b}}$, Ronald W. Davis ${ }^{\mathrm{b}, *}$ \\ and Anand K. Ramasubramanian ${ }^{\mathrm{a}, *}$ \\ ${ }^{a}$ Department of Chemical and Materials Engineering, San José State University, San José, CA, USA \\ ${ }^{\mathrm{b}}$ Stanford Genome Technology Center, Stanford University School of Medicine, Palo Alto, CA, USA
}

\begin{abstract}
.
BACKGROUND: Myalgic encephalomyelitis/Chronic Fatigue Syndrome (ME/CFS) is a poorly understood disease. Amongst others symptoms, the disease is associated with profound fatigue, cognitive dysfunction, sleep abnormalities, and other symptoms that are made worse by physical or mental exertion. While the etiology of the disease is still debated, evidence suggests oxidative damage to immune and hematological systems as one of the pathophysiological mechanisms of the disease. Since red blood cells (RBCs) are well-known scavengers of oxidative stress, and are critical in microvascular perfusion and tissue oxygenation, we hypothesized that RBC deformability is adversely affected in ME/CFS.

METHODS: We used a custom microfluidic platform and high-speed microscopy to assess the difference in deformability of RBCs obtained from ME/CFS patients and age-matched healthy controls.

RESULTS AND CONCLUSION: We observed from various measures of deformability that the RBCs isolated from ME/ CFS patients were significantly stiffer than those from healthy controls. Our observations suggest that RBC transport through microcapillaries may explain, at least in part, the ME/CFS phenotype, and promises to be a novel first-pass diagnostic test.
\end{abstract}

Keywords: Chronic Fatigue Syndrome, microfluidics, cell deformability, red blood cells

\section{Introduction}

Myalgic encephalomyelitis/Chronic Fatigue Syndrome (ME/CFS) is a multi-systemic, debilitating illness of unknown etiology affecting millions of individuals worldwide [1], with capacity to persist for decades. Although an abnormal profile of circulating proinflammatory cytokines, and the presence of chronic oxidative and nitrosative stresses have been identified and correlated with severity in ME/CFS $[2,3]$, there are no molecular or cellular biomarkers of the disease. Consequently, without any definitive or even non-specific diagnosis, the disease may be misdiagnosed or remain in disguise for a long time. Of interest, the shape and size of the red blood cells (RBC) change appreciably in response to oxidative and inflammatory stresses $[4,5]$. The usual shape of RBC is biconcave discoid, which provides a specific surface area-to-volume ratio that facilitates large reversible deformations to any arbitrary shape, enabling travel through microvessels for optimal tissue oxygenation [6-8]. The deformation of $\mathrm{RBCs}$ to physical forces has been investigated as a possible diagnostic biomarker for sickle cell disease [9], malaria [10], and Gulf War Illness [11]. In this study, we tested the hypothesis that deformability of RBCs is significantly altered in ME/CFS compared to normal population, using a high-throughput microfluidic device and high-speed microscopy [10].

\footnotetext{
*Corresponding authors: Anand K. Ramasubramanian, Ph.D., Department of Chemical and Materials Engineering, San José State University, CA 95192-0082, USA. Tel.: +1 408924 3922; E-mail: anand.ramasubramanian@ sjsu.edu and Ronald W. Davis, Ph.D., Stanford Genome Technology Center, Stanford University School of Medicine, Palo Alto, CA 94304, USA. Tel.: +1 650721 5614; E-mail: ron.davis@stanford.edu.
} 


\section{Materials and methods}

ME/CFS patients previously diagnosed by a physician using the Canadian Consensus Criteria [11] were selected for the study ( $n=16$ pairs of patients with age matched healthy controls). Written consent was obtained following Stanford IRB-40146. The mean ages were $52 \pm 7.7$ for the healthy controls (HC) and $52 \pm 6.7$ for the ME/CFS patients. The disability scores were $94 \pm 5.4 \%$ for the HC and $44 \pm 19 \%$ for the patients. The female to male ratio was 9:7 for the healthy group and 11:5 for the ME/CFS group. All experiments were performed within 3-6h of blood draw (all the time at room temperature), and the exact timing did not affect our results [12]. Whole blood was centrifuged at $250 \times \mathrm{G}$ for 20 minutes to obtain the RBC fraction. A dilute suspension ( $\sim 1 \%$ hematocrit) of $10^{7}$ $\mathrm{RBCs} / \mathrm{ml}$ in PBS (pH 7.4) was perfused through microfluidic channels using a custom vacuum-based delivery system at a pressure of -2 psi. The microfluidic channels were made of polydimethylsiloxane (PDMS) using Silicon master molds, as described previously in detail [10]. The channels were washed with $0.1 \%$ pluronic F127 (in PBS), before the cells were perfused, in order to prevent non-specific cell adhesion. RBCs moved through channels of progressively narrower cross section, starting from a width of $1 \mathrm{~mm}$ tapering down to $20 \mu \mathrm{m}$ before entering the $5 \mu \mathrm{m} \times 5 \mu \mathrm{m}$ test channels. The flow of RBCs through the $5 \mu \mathrm{m}$ test channels was visualized at $40 \times$ magnification and $4000 \mathrm{fps}$. The videos were analyzed off-line to quantify three parameters as a measure of deformability: entry time (time taken by the cells to fully enter the $5 \mu \mathrm{m}$ test channels); transit velocity (velocity of RBCs traveling a distance of $100 \mu \mathrm{m}$ through the test channels); and elongation index (ratio of the length of RBCs in the test channel to the length before entry into the test channel) (Fig. 1A).

\section{Results and discussion}

We first validated the ability of the microfluidic platform to detect changes in RBC deformability. RBCs from healthy volunteers were treated with $0.01 \%$ glutaraldehyde and perfused, since this treatment has been shown to have a cell stiffening effect $[13,14]$. Consistent with published studies, we observed that the cells show significantly higher entry time $(\sim 49 \%)$, lower transit velocity $(\sim 47 \%)$ and lower elongation index $(\sim 7 \%)$, as compared to untreated RBCs $(p<0.0001, n=3)$. Next, we perfused RBCs from healthy controls and CFS patients through the microfluidic channels. We observed that the diameter of RBCs prior to entering the test channels in ME/CFS patients was $\sim 10 \%$ more compared to $\mathrm{HC}$. The RBCs from ME/CFS patients showed a broader distribution compared to the HC, and a notable population of the RBCs $(\sim 20 \%)$ from ME/CFS patients were unusually bigger $(>12 \mu \mathrm{m})$, suggesting the varying impact of the disease on RBCs isolated from different patients (Fig. 1B). Next, we observed $\sim 15 \%$ decrease in the elongation index of RBCs from ME/CFS patients compared those from HC. The distribution of elongation indices shows that most of the cells (>70\%) from ME/CFS patients do not deform after entering the test channels, while majority of cells from HC deformed at least 10-25\% upon entering the test channels (Fig. 1C). By comparing the change in cell diameters normalized to the width of the test channel before $\left(L_{1} / d\right)$ and after $\left(L_{2} / d\right)$ entry, Fig. 1D clearly demonstrates that RBCs from ME/CFS patients deform 7-fold less compared to those from the HC. The cells from ME/CFS patients take $\sim 14 \%$ more time to enter the test channels (Fig. 1E), and upon entering the channels, travel $\sim 18 \%$ slower than those from HC (Fig. 1F). The distributions for entry time and transit velocity are skewed towards lower values for $\mathrm{HC}$ and towards higher values for those for ME/CFS patients. Because of the nature of the disease, often most patients were prescribed a wide range of medications and supplements depending on their particular symptoms and condition. For instance, these medications and supplements include Rituxan and loratadine or low-dose naltrexone, Vitamin D3 etc. Based on extensive literature search, we found only one of the drugs, colchicine, 
A

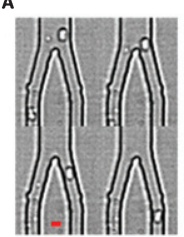

B1

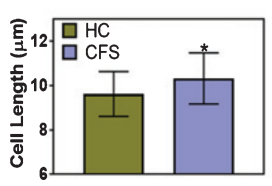

E1

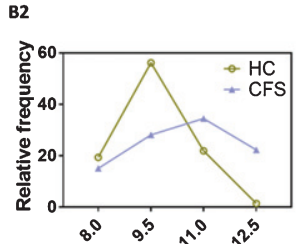

Cell Length $(\mu \mathrm{m})$

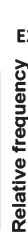

C1
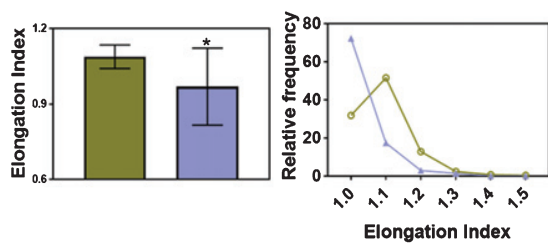

Elongation Index

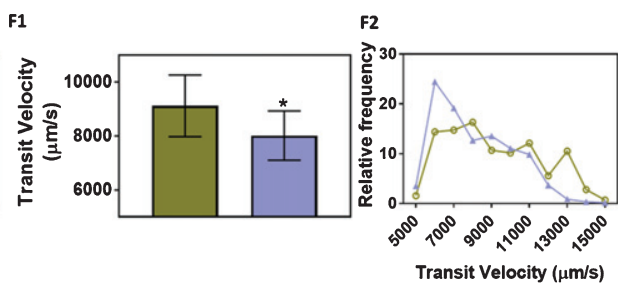

Fig. 1. Red blood cell (RBC) motion in microfluidic channels. (A) Representative traces of a RBC before, during and after entry through the test channels $(5 \mu \mathrm{m} \times 5 \mu \mathrm{m})$. Scale bar is $5 \mu \mathrm{m}$.; (B1) Cell length (major axis) and (B2) Distribution of cell lengths, of RBCs before entering the test channels; (C1) Elongation index and (C2) Distribution of elongation indices of deformed RBCs inside the test channels; (D) Comparison of scaled average lengths of undeformed (L1) and deformed (L2) RBCs from healthy controls and ME/CFS patients. The lengths were scaled to channel width $(\mathrm{d}=5 \mu \mathrm{m})$; (E1) Entry times and (E2) Distribution of entry times, i.e., time taken by RBCs to fully enter the test channels; (F1) Transit velocity and (F2) Distribution of transit velocities, based on the time taken by RBCs to travel $100 \mu \mathrm{m}$ distance inside the test channels. The results are based on at least two separate microfluidic devices per donor, and at least 100 cells were tested per device. The results are shown as mean $\pm \mathrm{SD}$. *denotes significant differences in comparison to healthy samples, based on 2-way ANOVA with Tukey's post hoc analysis ( $p<0.0001 ; n=16$ pairs). For distribution, the results are presented as histogram with relative frequency in percentage. HC: RBCs from healthy donors; CFS: RBCs from ME/CFS patients.

which was prescribed to one patient, has been shown to slightly affect cellular deformability $[15,16]$. However, the deformability parameters for this particular patient were well within the range for other patients. The patients and volunteers identified themselves as non-smokers [17].

Together, the various estimates show that the RBCs in ME/CFS patients are significantly less deformable than those of HC. Our observations may provide an opportunity for a first-pass diagnostic test for ME/CFS with a finger-prick blood sample, with a caveat that the test may be non-specific as other inflammatory conditions or existing co-morbidities may alter RBC deformability, and may have to be placed in context with other clinical presentations. We speculate that the larger and less deformable RBCs in ME/CFS patients may partly explain the musculoskeletal pain and fatigue in the pathophysiology of ME/CFS due to impaired microvascular perfusion and tissue oxygenation. Of note, it was previously reported that no significant difference in average deformability was observed between erythrocytes obtained from healthy controls and ME/CFS patients, as measured by ektacytometer [18]. This apparent contradiction can be because of the lower sensitivity of the ektacytometer, which reports the deformability based on time and population averages indirectly from light scattering, while as seen from this work, the deformability is best measured directly on an individual cell basis.

\section{Acknowledgments}

The authors would like to express their heartfelt appreciation to numerous ME/CFS patients and their families for access to blood for improving the understanding and treatment of the disease. The authors would like to thank Dr. Liat Rosenfeld for granting access to the high-speed camera used to image cell movements through microchannels, and Ms. Jaime Seltzer for making useful comments on 
proofreading of the manuscript. The authors would also like to thank the Open Medicine Foundation for financial support.

\section{Authorship contributions}

A.K.S., M.N.G., R.W.D., and A.K.R designed the study, analyzed the data and wrote the manuscript. J.W. arranged and collected blood samples from ME/CFS patients, and the matching controls. A.K.S., B.R.S., A.A., V.N., and J.K.D performed microfluidics experiments.

\section{Disclosure of conflicts of interest}

The authors declare no conflict of interest.

\section{References}

[1] Clayton EW. Beyond myalgic encephalomyelitis/chronic fatigue syndrome: An IOM report on redefining an illness. JAMA. 2015;313(11):1101-2.

[2] Montoya, J.G., et al., Cytokine signature associated with disease severity in chronic fatigue syndrome patients. Proc Natl Acad Sci U S A. 2017;114(34):E7150-8.

[3] Gerwyn M, Maes M. Mechanisms explaining muscle fatigue and muscle pain in patients with myalgic encephalomyelitis/chronic fatigue syndrome (ME/CFS): A review of recent findings. Curr Rheumatol Rep. 2017;19(1):1.

[4] Richards RS, Wang L, Jelinek H. Erythrocyte oxidative damage in chronic fatigue syndrome. Arch Med Res. 2007;38(1):94-8.

[5] Pretorius E. Erythrocyte deformability and eryptosis during inflammation, and impaired blood rheology. Clin Hemorheol Microcirc. 2018;69(4):545-50.

[6] Kim J, Lee H, Shin S. Advances in the measurement of red blood cell deformability: A brief review. Journal of Cellular Biotechnology. 2015;1(1):17.

[7] Connes P, et al. Blood rheological abnormalities in sickle cell anemia. Clin Hemorheol Microcirc. 2018;68(2-3):165-72.

[8] Driessen GK, et al. Effect of reduced red cell "deformability" on flow velocity in capillaries of rat mesentery. Pflugers Arch. 1980;388(1):75-8.

[9] Alapan Y, et al. Dynamic deformability of sickle red blood cells in microphysiological flow. Technology (Singap World Sci). 2016;4(2):71-9.

[10] Saha AK, et al. Cellular cholesterol regulates monocyte deformation. J Biomech. 2017;52:83-8.

[11] David S, Bell M. The Doctor's Guide to Chronic Fatigue Syndrome, pp. 122-4.

[12] Baskurt OK, et al. New guidelines for hemorheological laboratory techniques. Clin Hemorheol Microcirc. 2009;42(2): 75-97.

[13] Holmes D, et al. Separation of blood cells with differing deformability using deterministic lateral displacement(dagger). Interface Focus. 2014;4(6):20140011.

[14] Corry WD, Meiselman HJ. Modification of erythrocyte physicochemical properties by millimolar concentrations of glutaraldehyde. Blood Cells. 1978;4(3):465-83.

[15] Tsai MA, Waugh RE, Keng PC. Passive mechanical behavior of human neutrophils: Effects of colchicine and paclitaxel. Biophys J. 1998;74(6):3282-91.

[16] Chien S, Sung KL. Effect of colchicine on viscoelastic properties of neutrophils. Biophys J. 1984;46(3):383-6.

[17] Salbas K. Effect of acute smoking on red blood cell deformability in healthy young and elderly non-smokers, and effect of verapamil on age- and acute smoking-induced change in red blood cell deformability. Scand J Clin Lab Invest. 1994;54(6):411-6.

[18] Brenu EW, et al., Immune and hemorheological changes in chronic fatigue syndrome. J Transl Med. $2010 ; 8: 1$. 